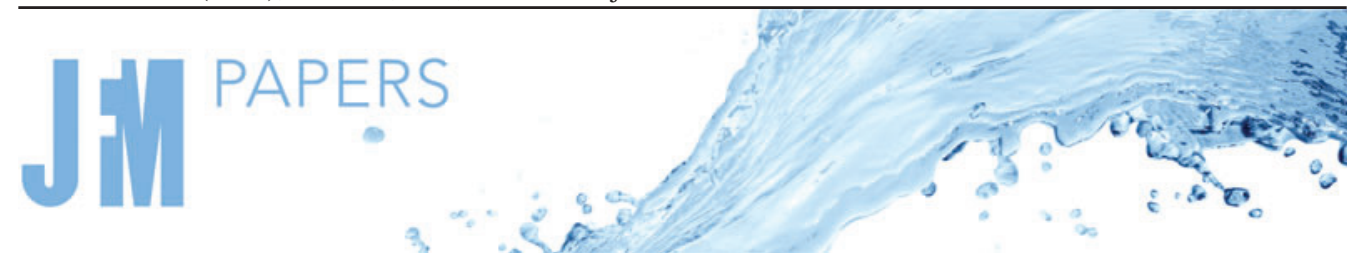

\title{
Asymptotic behaviour at the wall in compressible turbulent channels
}

\author{
Akanksha Baranwal $^{1}$, Diego A. Donzis ${ }^{1, \dagger}$ and Rodney D.W. Bowersox ${ }^{1}$ \\ ${ }^{1}$ Department of Aerospace Engineering, Texas A\&M University, College Station, TX 77843, USA
}

(Received 2 April 2021; revised 22 August 2021; accepted 30 November 2021)

The asymptotic behaviour of Reynolds stresses close to walls is well established in incompressible flows owing to the constraint imposed by the solenoidal nature of the velocity field. For compressible flows, thus, one may expect a different asymptotic behaviour, which has indeed been noted in the literature. However, the transition from incompressible to compressible scaling, as well as the limiting behaviour for the latter, is largely unknown. Thus, we investigate the effects of compressibility on the near-wall, asymptotic behaviour of turbulent fluxes using a large direct numerical simulation (DNS) database of turbulent channel flow at higher than usual wall-normal resolutions. We vary the Mach number at a constant friction Reynolds number to directly assess compressibility effects. We observe that the near-wall asymptotic behaviour for compressible turbulent flow is different from the corresponding incompressible flow even if the mean density variations are taken into account and semi-local scalings are used. For Mach numbers near the incompressible regimes, the near-wall asymptotic behaviour follows the well-known theoretical behaviour. When the Mach number is increased, turbulent fluxes containing wall-normal components show a decrease in the slope owing to increased dilatation effects. We observe that $R_{v v}$ approaches its high-Mach-number asymptote at a lower Mach number than that required for the other fluxes. We also introduce a transition distance from the wall at which turbulent fluxes exhibit a change in scaling exponents. Implications for wall models are briefly presented.

Key words: compressible turbulence, turbulence simulation, turbulent boundary layers

\section{Introduction}

The understanding of wall-bounded flows is of paramount importance in the design of high-speed vehicles. The estimation of thermal and viscous loads on aircraft requires

$\dagger$ Email address for correspondence: donzis@tamu.edu

(C) The Author(s), 2021. Published by Cambridge University Press. This is an Open Access article, distributed under the terms of the Creative Commons Attribution licence (https://creativecommons. org/licenses/by/4.0/), which permits unrestricted re-use, distribution, and reproduction in any medium, provided the original work is properly cited. 


\section{A. Baranwal, D.A. Donzis and R.D.W. Bowersox}

robust turbulence models which can predict accurately turbulent statistics close to the wall. While extremely complicated, significant advances have been made to understand the details of these flows, in part, owing to the availability of large computational resources that generate massive datasets for examination. This has been particularly so for incompressible flows. Because of the additional complexity, larger parameter space and much greater computational cost, less is known about their compressible counterparts.

The incompressible limit is commonly considered as a reference point around which changes in turbulence statistics are quantified as compressibility levels are increased. This is, in part, owing to the large body of literature on the behaviour of, for example, Reynolds stresses and heat flux close to the wall in incompressible flows that has developed over many decades (e.g. Hinze 1975; Lai \& So 1990; So et al. 1991a; So, Zhang \& Speziale $1991 b$; Durbin 1993 and subsequent work). Work examining the similarities with the near-wall behaviour in compressible flows followed (e.g. Zhang et al. 1992; Sommer, So \& Zhang 1993; So, Gatski \& Sommer 1998). It is a common practice, for example, to extend incompressible turbulence models to compressible situations by noting that the governing equations, when Favre averaged, share some commonalities with the incompressible Navier-Stokes equations. A number of classical compressibility corrections are based on Morkovin's hypothesis, which states that compressibility effects mainly influence turbulence statistics through variations in mean density. In this approximation, density fluctuations have a negligible effect. A number of studies in the past have focused on compressibility effects and assessing the validity of Morkovin's hypothesis using data from direct numerical simulations (DNSs) in different configurations (e.g. Coleman, Kim \& Moser 1995; Duan, Beekman \& Martin 2011; Smits \& Dussauge 2006 and references therein). There is also the complexity associated with different possible normalizations, among which we can mention wall, semi-local and outer scaling. In general, studies have found a much better collapse of the Reynolds stresses with semi-local normalization (Patel et al. 2015; Modesti \& Pirozzoli 2016; Trettel \& Larsson 2016). However, Morinishi, Tamano \& Nakabayashi (2004) noted, using their own data as well as those from Coleman et al. (1995), that Reynolds stresses exhibit a change in slope close to the wall with Mach number. This observation does not seem to conform to Morkovin's proposal. Perhaps more importantly, this asymptotic behaviour, which is distinct from the well-known incompressible laws close to the wall, has only been noted but has not been studied systematically. For example, it is not known how Reynolds stresses or turbulent heat fluxes scale in general close to the wall in compressible flows, or how the scaling changes with compressibility, or the way in which they transition from incompressible to compressible conditions. Knowing the correct asymptotic behaviour close to the wall is important not only for fundamental understanding but also for turbulence modelling as one expects accurate models to satisfy the exact analytical behaviour close to the wall (Bowersox 2009). This is the thrust of the present work. We investigate compressibility effects on turbulent fluxes close to the wall by carrying out highly resolved direct numerical numerical simulations of turbulent channels with bulk Mach numbers from 0.2 to 3.1. This range allows us to identify near-wall asymptotic scaling laws for several turbulent fluxes, Mach number effects and transitions from incompressible to compressible conditions.

\section{Direct numerical simulations}

The governing equations expressing conservation of mass, momentum and energy for a compressible flow are given by

$$
\frac{\partial \rho}{\partial t}+\frac{\partial\left(\rho u_{i}\right)}{\partial x_{i}}=0,
$$


Wall asymptotics in compressible turbulent channels

$$
\begin{gathered}
\frac{\partial\left(\rho u_{i}\right)}{\partial t}+\frac{\partial\left(\rho u_{i} u_{j}\right)}{\partial x_{j}}=-\frac{\partial p}{\partial x_{i}}+\frac{\partial \sigma_{i j}}{\partial x_{j}}+\rho f_{i} \delta_{i 1}, \\
\frac{\partial(\rho e)}{\partial t}+\frac{\partial\left(\rho e u_{j}\right)}{\partial x_{j}}=-p \frac{\partial u_{j}}{\partial x_{j}}+\frac{\partial}{\partial x_{j}}\left(\kappa \frac{\partial T}{\partial x_{j}}\right)+\sigma_{i j} s_{i j},
\end{gathered}
$$

where $\rho$ is the fluid density, $u_{i}$ the $i$ th component of the velocity, $p$ the pressure, $e$ the internal energy per unit mass and $T$ the temperature. The viscous stress is given by $\sigma_{i j}=\mu\left(\partial u_{i} / \partial x_{j}+\partial u_{j} / \partial x_{i}-\frac{2}{3} \delta_{i j}\left(\partial u_{k} / \partial x_{k}\right)\right)$ and the strain rate tensor is given by $s_{i j}=\frac{1}{2}\left(\partial u_{i} / \partial x_{j}+\partial u_{j} / \partial x_{i}\right)$. The molecular viscosity, $\mu$, obeys a power law of the form of $T^{a}$ where $a=0.5$. The thermal conductivity, $\kappa$, is related to $\mu$ through $\kappa=\mu C_{p} / P_{r}$ with $P_{r}=0.7$. To close the system of equations, the ideal-gas equation of state $p=\rho R T$ is assumed. A body force $\rho f_{1}$ is added in the streamwise momentum equation thus ensuring a constant mass flow rate. Bulk density is also kept constant at every time step. This approach, which has been extensively used in the literature (e.g. Coleman et al. 1995; Huang, Coleman \& Bradshaw 1995; Foysi, Sarkar \& Friedrich 2004; Morinishi et al. 2004; Gerolymos \& Vallet 2014; Sciacovelli, Cinnella \& Gloerfelt 2017) allows us to accurately determine simulation conditions a priori. The equations are discretized on a mesh that is uniform in the streamwise $(x)$ and spanwise $(z)$ directions. The grid in the wall-normal $(y)$ direction follows a hyperbolic tangent stretching function. The spatial derivatives in the $x$ and $z$ directions are computed using sixth-order compact schemes. For the wall-normal direction, we also use a sixth-order compact scheme in interior points which is reduced to fourth- and third-order accurate compact schemes at the last two grid points in the domain. The variables are marched in time using a third-order low-storage Runge-Kutta scheme. Periodic boundary conditions are applied in both streamwise and spanwise directions. At the walls, we apply no-slip, isothermal boundary conditions. The boundary condition for pressure is obtained by evaluating the $y$-momentum equation at the wall:

$$
\frac{\partial p}{\partial y}=\frac{\partial \sigma_{y x_{j}}}{\partial x_{j}}
$$

which, in addition to represent the proper Navier-Stokes physics at the physical boundary, was found to be much more stable numerically than the commonly used zero-pressure-gradient condition at the wall. A Courant-Friedrichs-Lewy (CFL) condition was used to determine the time step size which, for all situations in the database, was between 0.2 and 0.4. This is smaller than values commonly used in the literature and ensured stable simulations free of spurious fluctuations.

Using standard notation, the bulk, wall and centreline values of a variable $f$ are denoted by $f_{b}, f_{w}$ and $f_{c}$, respectively. Reynolds and Favre decompositions are denoted by $\bar{q}+q^{\prime}$ and $\tilde{q}+q^{\prime \prime}$, respectively. The averages in these decompositions are taken along the homogeneous directions (i.e. $x-z$ planes) and time. We found that the longest variations for statistics of interest here are of order $h / \overline{u_{b}}$, where $h$ is the channel half-width. As expected, this time scale is commensurate with the eddy-turnover time of the turbulence in the centre of the channel and thus representative of the largest structures. To ensure statistical independence, our temporal averages are done for snapshots separated by intervals of $5 h / \overline{u_{b}}$. Good statistical convergence was achieved with 20-30 time snapshots. Our temporal averages involved 50 snapshots for velocity, density and temperature fields.

The bulk and friction Reynolds numbers are $R e_{b} \equiv \overline{\rho_{b}} \overline{u_{b}} h / \overline{\mu_{w}}$ and $R e_{\tau}=\overline{\rho_{w}} u_{\tau} h / \overline{\mu_{w}}$, respectively, with $u_{\tau} \equiv \sqrt{\tau_{w} / \rho_{w}}$ being the friction velocity. The bulk and centreline Mach numbers are $M_{b} \equiv \overline{u_{b}} / \sqrt{\gamma R T_{w}}$ and $M_{c} \equiv \overline{u_{c}} / \sqrt{\gamma R T_{c}}$, respectively. The friction 


\begin{tabular}{rrrrrrrrrrrrrrr}
\hline$M_{b}$ & $R e_{b}$ & $M_{c}$ & $R e_{c}$ & $R e_{\tau}$ & $R e_{\tau}^{*}$ & $\Delta y_{\min }^{+}$ & $\Delta y_{\max }^{+}$ & $\Delta x^{+}$ & $\Delta z^{+}$ & $\left.\frac{\Delta x}{\eta}\right|_{\max }$ & $\left.\frac{\Delta z}{\eta}\right|_{\max }$ & Line style \\
0.2 & 4966 & 0.23 & 5692 & 295 & 293 & 0.08 & 2.9 & 14.5 & 4.8 & 9.7 & 3.2 & - \\
0.3 & 4928 & 0.35 & 5638 & 294 & 289 & 0.08 & 2.9 & 14.4 & 4.8 & 9.7 & 3.2 & - \\
0.4 & 4902 & 0.46 & 5582 & 294 & 286 & 0.08 & 2.9 & 14.4 & 4.8 & 9.6 & 3.2 & - \\
0.5 & 4854 & 0.57 & 5476 & 293 & 281 & 0.05 & 3.2 & 14.4 & 4.8 & 9.6 & 3.2 & - \\
0.6 & 4934 & 0.68 & 5498 & 301 & 283 & 0.05 & 3.3 & 14.8 & 4.9 & 9.9 & 3.3 & - \\
0.8 & 4937 & 0.89 & 5371 & 307 & 276 & 0.05 & 3.4 & 15.1 & 5.0 & 10.0 & 3.3 & - \\
1.2 & 4927 & 1.26 & 5022 & 325 & 259 & 0.05 & 3.6 & 15.9 & 5.3 & 10.5 & 3.5 & --- \\
1.5 & 5752 & 1.50 & 5489 & 393 & 277 & 0.10 & 4.0 & 19.3 & 6.4 & 12.7 & 4.2 & --- \\
2.4 & 7149 & 1.98 & 5631 & 572 & 279 & 0.10 & 6.2 & 14.0 & 4.7 & 9.2 & 3.1 & ---
\end{tabular}

Table 1. Details of DNS flow conditions and grid resolutions.

Reynolds number, based on centreline viscosity and density, $R e_{\tau}^{*} \equiv \overline{\rho_{c}}\left(\tau_{w} / \overline{\rho_{c}}\right)^{1 / 2} h / \overline{\mu_{c}}$, is kept approximately constant across all of the cases to directly assess compressibility effects (Trettel \& Larsson 2016). Table 1 summarizes the important parameters for the DNS database used here. We note that the near-wall resolution is very high with the first grid point located at $y^{+}$as low as 0.05 , which is much better than that commonly used in the literature. As we show below, these very high resolutions are needed to capture the correct asymptotic behaviour at the wall.

Because of the periodic boundary conditions in the streamwise and spanwise directions, one needs to make sure that the length of the domain is enough to capture structures in that direction. Thus, our domain has dimensions $4 \pi h \times 2 h \times 4 \pi / 3 h$, which is larger than that widely used in the literature (e.g. Trettel \& Larsson 2016; Yu, Xu \& Pirozzoli 2019). Furthermore, we have verified that two-point streamwise and spanwise correlation functions decay to small values inside the domain in a manner completely consistent with the literature (e.g. Wu \& Martin 2007; Li et al. 2019). Finally, as a direct assessment of boundary condition effects on the quantities studied here, we have run additional simulations with a domain which is $20 \%$ shorter and confirmed that the near-wall asymptotic results are unaffected.

Simulations with $R e_{\tau} \leqslant 393$ have grid resolutions $256 \times 512 \times 256$, while simulations with $R e_{\tau}=572$ and 745 have resolutions $512 \times 512 \times 512$ and $640 \times 512 \times 512$, respectively. Some basic first- and second-order statistics at $R_{b}=3000$ and $M_{b}=1.5$ are shown in figure 1, where we see excellent agreement between our results and those of Coleman et al. (1995) at the same conditions. Detailed grid-convergence studies have been performed. In figure 2 , we show results for the largest $R e_{\tau}$ and $M_{c}$ case in our database, which corresponds to the most challenging condition. The related details are provided in table 2. All quantities of interest collapse for cases C, D and E close to the wall. It is interesting to note that the wall-normal turbulent stress converges at a higher resolution than that required for the mean velocity and other turbulent fluxes and thus imposes a stricter resolution criterion. As noted in table 1, our database consists of simulations with resolutions in $x, y$ and $z$ comparable to the resolutions corresponding to case $\mathrm{D}$ of our convergence study and are, therefore, converged for this study. The resolutions in $x$ and $z$ are also commensurate with the DNS resolutions used in the literature while wall-normal grid resolutions are larger than the usual. 
(a)

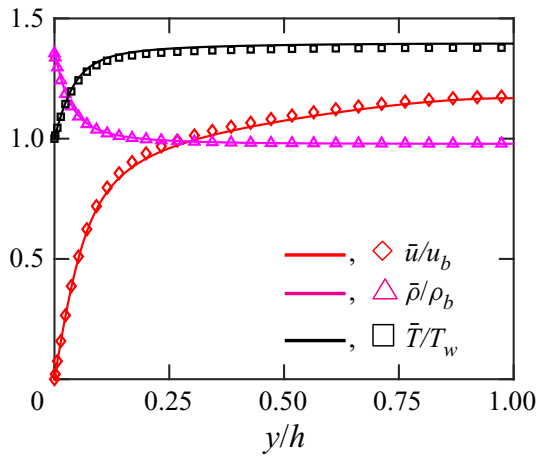

(b)

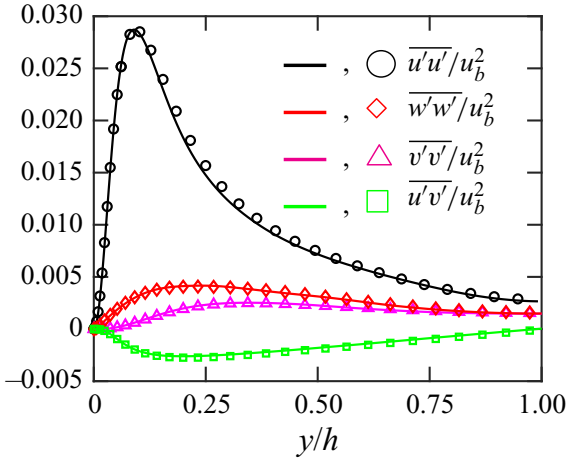

Figure 1. Validation for the channel at $R_{b}=3000$ and $M_{b}=1.5$. Solid lines, present DNS results; symbols, data from Coleman et al. (1995).

$\begin{array}{lrrrrrrrrrr}\text { Case } & N_{x} & N_{y} & N_{z} & \Delta y_{\min }^{+} & \Delta y_{\max }^{+} & \Delta x^{+} & \Delta z^{+} & \left.\frac{\Delta x}{\eta}\right|_{\max } & \left.\frac{\Delta z}{\eta}\right|_{\max } & \text { Marker } \\ \mathrm{A} & 256 & 256 & 256 & 0.24 & 16.6 & 36.5 & 12.2 & 23.9 & 7.9 & \triangleleft \\ \mathrm{B} & 256 & 512 & 256 & 0.09 & 8.8 & 36.7 & 12.2 & 24.0 & 8.0 & \bigcirc \\ \mathrm{C} & 512 & 512 & 512 & 0.09 & 8.8 & 18.3 & 6.1 & 12.0 & 4.0 & \diamond \\ \mathrm{D} & 640 & 512 & 512 & 0.09 & 8.8 & 14.8 & 6.1 & 9.7 & 4.0 & \triangle \\ \mathrm{E} & 1024 & 512 & 512 & 0.087 & 8.73 & 9.1 & 6.1 & 5.9 & 3.94 & \square\end{array}$

Table 2. Simulation details for convergence study of a case with $R e_{\tau}=745$ and $M_{c}=2.22$.

\section{Second-order statistics}

A number of studies (Huang et al. 1995; Foysi et al. 2004; Morinishi et al. 2004; Trettel \& Larsson 2016) noted that the use of the semi-local transformed coordinate, $y^{*} \equiv \bar{\rho} \sqrt{\tau_{w} / \bar{\rho}} h / \bar{\mu}$, as well as Morkovin's hypothesis leads to better collapse of Reynolds stresses in compressible flows with their incompressible counterparts. Morkovin-scaled Reynolds stresses are given by $R_{i j}^{*} \equiv \bar{\rho} \widetilde{u}_{i}^{\pi \prime} u_{j}^{\prime \prime} / \tau_{w}$. Similarly, the temperature flux can be scaled as $R_{v T}^{*}=\bar{\rho} \widetilde{v^{\prime \prime} T^{\prime \prime}} /\left(\rho_{w} u_{\tau} T_{\tau}\right)$, where $T_{\tau} \equiv-\lambda(\partial \bar{T} / \partial y)_{w} /\left(\bar{\rho}_{w} c_{p} u_{\tau}\right)$ is the friction temperature. In figure $3(a-e)$, we show $R_{i j}^{*}$ and $R_{v T}^{*}$ against $y^{*}$ for all the cases in our database. An excellent collapse is observed for $R_{u u}^{*}$ for all Mach numbers close to the wall. This degree of universality, however, is not observed for other fluxes in the immediate vicinity of the wall. In figure $3(b-d)$, we can see three important features. First, we see that, close to the wall, the slope of the different fluxes changes systematically with Mach number. Second, this slope seems to transition to another scaling range with much weaker Mach number effects. This transition was also observed by Morinishi et al. (2004). And third, the transition between these two ranges appears to move further away from the wall at higher Mach numbers. Thus, it is clear that compressibility effects play a role in the asymptotic behaviour of turbulent fluxes as one approaches the wall. This is the main thrust of the rest of this work.

Consider a Taylor series of the fluctuating component of the velocity field at the wall:

$$
u^{\prime}=a_{u}+b_{u} y+c_{u} y^{2}+\ldots, \quad v^{\prime}=a_{v}+b_{v} y+c_{v} y^{2}+\ldots, \quad w^{\prime}=a_{w}+b_{w} y+c_{w} y^{2}+\ldots
$$



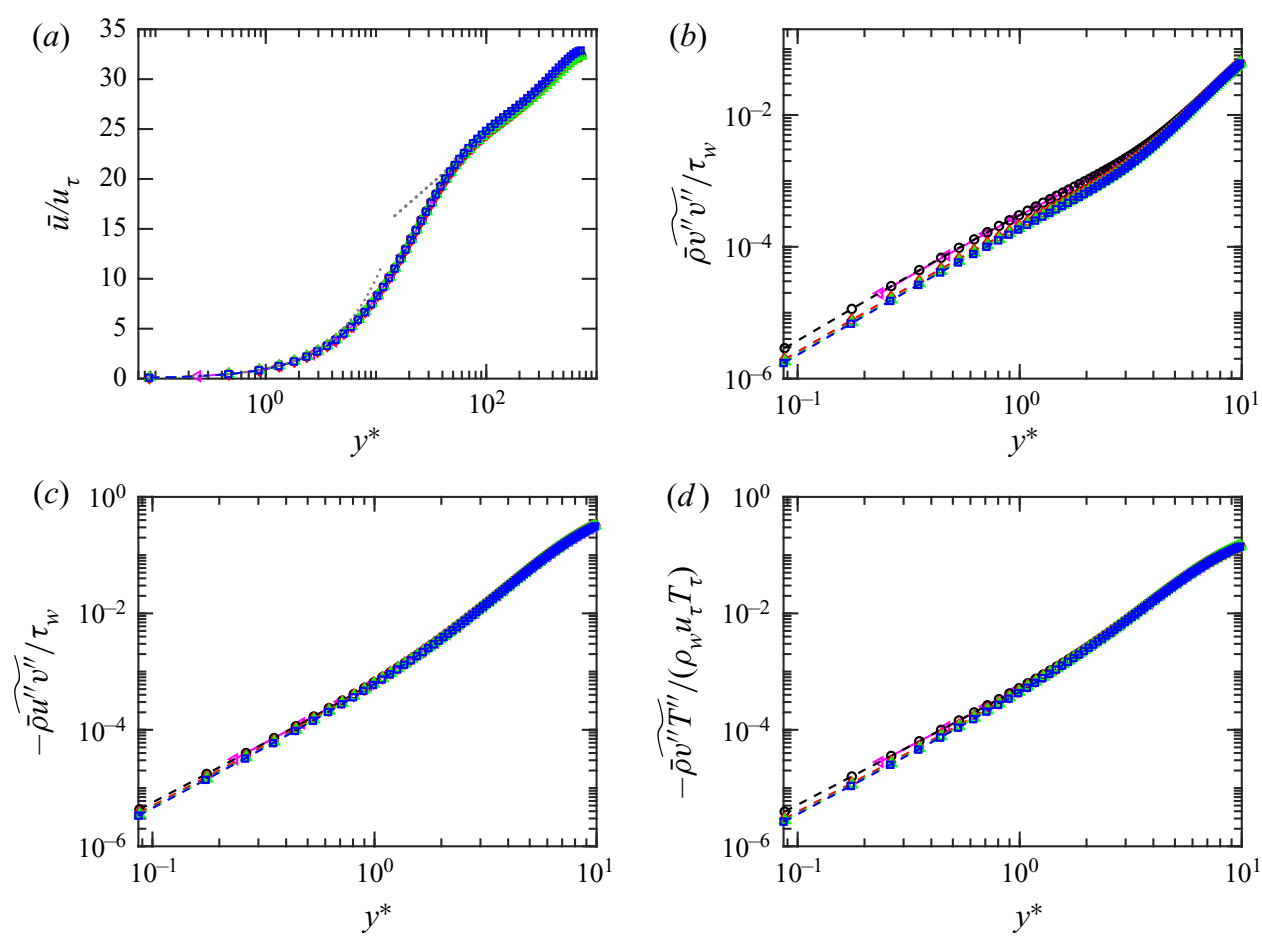

Figure 2. Grid convergence study for $R e_{b}=8301$ and $M_{b}=3$.1. In panel (a), dotted grey lines correspond to viscous and log-layer scalings, for reference. Symbols as in table 2.

The coefficients $a_{\alpha}$ (where $\alpha=u, v$ or $w$ ) are identically zero owing to the no-slip boundary condition at the wall. The other coefficients are given by $b_{u}=\partial u^{\prime} / \partial y$ and $c_{u}=(1 / 2) \partial^{2} u^{\prime} / \partial y^{2}$, and similarly for $v$ and $w$. If the flow is incompressible (solenoidal), mass conservation combined with the no-slip condition at the wall leads to an additional constraint in the wall-normal velocity component, namely, $\partial v^{\prime} / \partial y=b_{v}=0$. By taking the product between the expansions of different components and averaging, one finds the following well-known quadratic asymptotic laws for the streamwise and spanwise stresses: $\overline{u^{\prime} u^{\prime}} \approx \sigma_{u u}^{I} y^{2}$ and $\overline{w^{\prime} w^{\prime}} \approx \sigma_{w w}^{I} y^{2}$, with $\sigma_{u u}^{I}=\overline{b_{u} b_{u}}$ and $\sigma_{w w}^{I}=\overline{b_{w} b_{w}}$ (superscript $I$ denotes incompressible conditions). The wall-normal stress, however, presents a steeper quadratic growth: $\overline{v^{\prime} v^{\prime}} \approx \sigma_{v v}^{I} y^{4}$, with $\sigma_{v v}^{I}=\overline{c_{v} c_{v}}$. The Reynolds shear stress is similarly found to scale as $\overline{u^{\prime} v^{\prime}} \approx \sigma_{u v}^{I} y^{3}$ with $\sigma_{u v}^{I}=\overline{b_{u} c_{v}}$. For isothermal walls, there is no additional constraint on the Taylor expansion of fluctuating temperature and, thus, the wall-normal heat flux is $\overline{v^{\prime} T^{\prime}} \approx \sigma_{v T}^{I} y^{3}$ with $\sigma_{v T}^{I}=\overline{c_{v} b_{T}}$. When normalized with the friction velocity $u_{\tau}$, these stresses and fluxes will be denoted by $R_{\alpha \beta}$ (e.g. $R_{u u}=\overline{u^{\prime} u^{\prime}} / u_{\tau}^{2}$ ).

If, however, the flow is non-solenoidal, then $b_{v}$ does not vanish. Stresses involving wall-normal fluctuations $v^{\prime}$, then, will change their asymptotic behaviour because $v^{\prime} \sim y$ instead of $y^{2}$. The resulting scaling laws for both solenoidal and non-solenoidal conditions are summarized in table 3. Morinishi et al. (2004) also noted this theoretical asymptotic scaling law, $\overline{v^{\prime} v^{\prime}} \sim y^{2}$ in their compressible channel at $M_{c}=1.5$. As we see in figure 5, our data also exhibit power law behaviour and, thus, it is natural to investigate the behaviour of the scaling exponent in the functional form $R_{\alpha \beta} \approx \sigma_{\alpha \beta} y^{\gamma_{\alpha \beta}}$. Here, and in what follows, 
Wall asymptotics in compressible turbulent channels
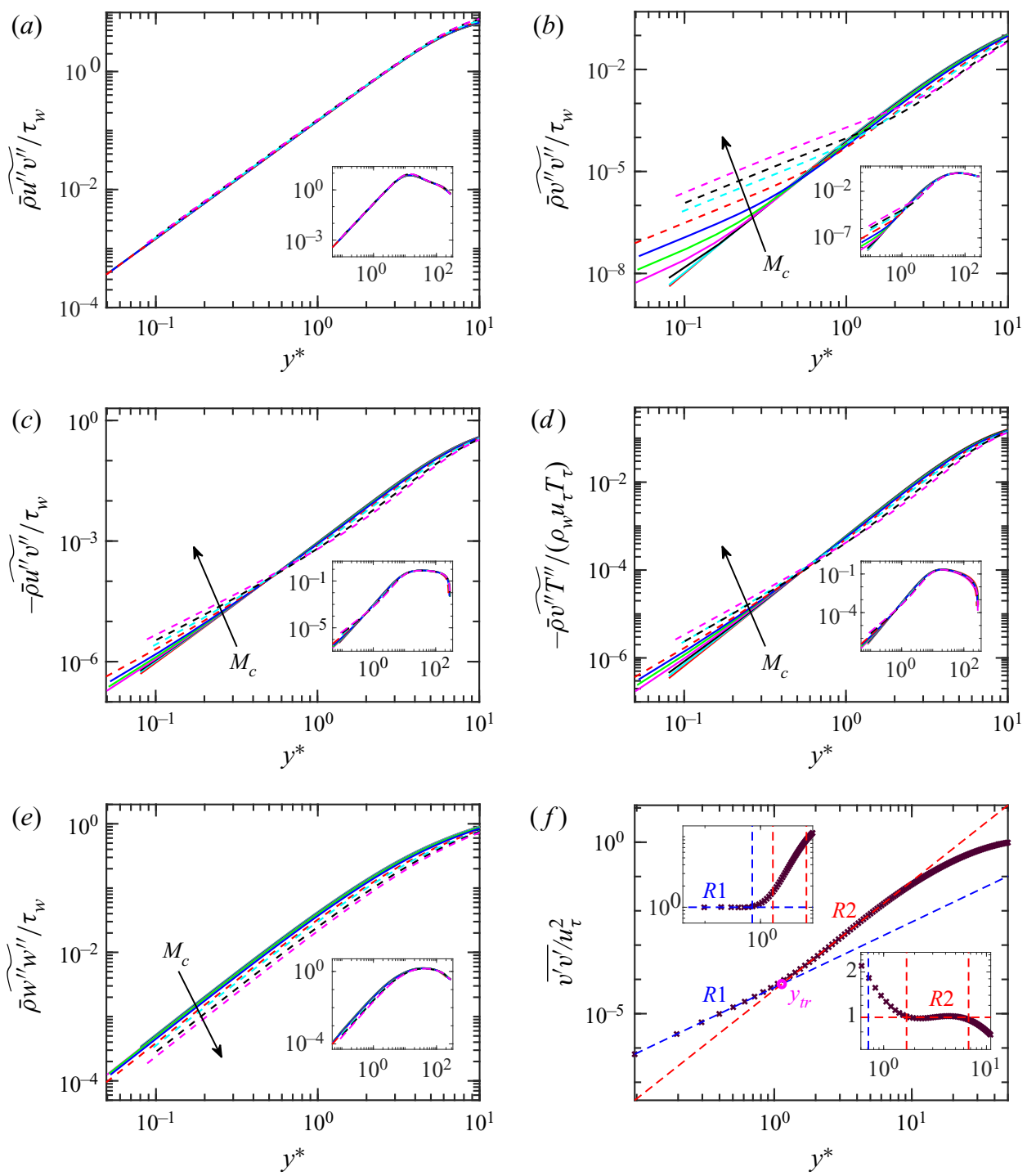

Figure 3. $(a-e)$ Density-scaled turbulent-flux distributions versus semi-local wall-normal coordinate. Colours as in table 1. Insets show same profiles up to $y^{*} \approx 300$. ( $f$ ) Fitting power laws to $R_{v v}$ for $M_{c}=1.5$. See text for inset description.

$R_{\alpha \beta}$ will denote a general stress where $\alpha$ and $\beta$ can be velocity components or temperature. Clearly, the prefactors $\sigma_{\alpha \beta}$ involving the $v$ component will also use $b_{v}$ instead of $c_{v}$ for non-solenoidal fields (e.g. for non-solenoidal conditions $\sigma_{v v}=\overline{b_{v} b_{v}}$ ). Of particular interest would be the variation of $\sigma_{\alpha \beta}$ and $\gamma_{\alpha \beta}$ with compressibility levels or Mach number.

Using well-resolved data such as those shown in figure $3(a-e)$, we have fit power laws in the two regions mentioned above, which are denoted by $R 1$ and $R 2$. Note that in simulations with insufficient wall resolution, what appears to be asymptotic behaviour may be, in fact, $R 2$. Thus, we also obtain fits in this regime for comparison purposes. The fitting procedure is shown in figure $3(f)$. To select the scaling regime, we compensated the Reynolds stresses by $y^{\gamma_{\alpha \beta}}$ and adjusted the limits such that the range over which a plateau 


$\begin{array}{lccccc} & \overline{u^{\prime} u^{\prime}} & \overline{v^{\prime} v^{\prime}} & \overline{w^{\prime} w^{\prime}} & \overline{u^{\prime} v^{\prime}} & \overline{v^{\prime} T^{\prime}} \\ \text { Solenoidal } & 2 & 4 & 2 & 3 & 3 \\ \text { Non-solenoidal } & 2 & 2 & 2 & 2 & 2\end{array}$

Table 3. Exponents $\gamma_{\alpha \beta}$ for near-wall asymptotic behaviour for $R_{\alpha \beta}(\alpha$ and $\beta$ are $u, v, w$ or $T$ ).

is observed (where a power law exists) is maximized. The result is illustrated in the two insets of figure $3(f)$, where the vertical dashed blue line marks the end of the fitting range for $R 1$ and vertical dashed red lines mark the fitting range for $R 2$. The corresponding fits are shown as dashed lines, blue and red for $R 1$ and $R 2$, respectively, in the main figure. This procedure was performed for both Reynolds and Favre-averaged stresses (e.g. $\overline{u^{\prime} v^{\prime}} / u_{\tau}^{2}$ and $\bar{\rho} u^{\prime \prime} v^{\prime \prime} / \tau_{w}$, respectively) with similar degrees of accuracy. The results, which quantify the compressibility effects noted qualitatively in figure 3 , are shown in figure 4 .

In figure $4(a)$, we see the exponent $\gamma_{v v}$ for both wall $\left(R_{v v}\right.$ versus $\left.y^{+}\right)$and semi-local $\left(R_{v v}^{*}\right.$ versus $\left.y^{*}\right)$ normalizations as a function of the centreline Mach number. The blue curves correspond to $R 1$ for which the wall and semi-local results are indistinguishable from each other. At low $M_{c}$, we see $\gamma_{v v} \approx 4$ as expected for solenoidal fields (table 3 ). As $M_{c}$ increases, $\gamma_{v v}$ deceases monotonically to approach values very close to 2 . This is the result of the emergence of dilatational motions which, at the wall, implies $b_{v} \neq 0$. We do note, though, that relaxing the solenoidal constraint simply implies that $\partial v^{\prime} / \partial y$ (i.e. $b_{v}$ ) is not bound to be zero; it does not imply that it must be non-zero. However, as already noted, the data do suggest strong compressibility effects even in $R 1\left(y^{+} \lesssim O(1)\right)$. This is interesting given that close to the wall, the velocity is the lowest and compressibility effects may be, in principle, expected to be weak. The drastic change in behaviour at the wall, thus, suggest a stronger coupling between turbulence close to and far from the wall.

The transition for $\gamma_{v v}$ starts at $M_{c} \approx 0.3$ and approaches its asymptote at $M_{c}$ somewhat below unity. It is interesting that the transition is not sharp and intermediate values $(2<$ $\left.\gamma_{v v}<4\right)$ are observed at a range of $M_{c}$. This suggests complex dynamics very close to the wall, as a simple Taylor expansion would suggest 2, 3 or 4 , with the prefactors determining which term is the dominant one. In figure 4(b), we compare the normalized prefactors $\sigma_{v v}$ obtained from the fits against the analytical prefactors $\overline{b_{v}^{2}} v_{w}^{2} / u_{\tau}^{4}=\overline{\left(\partial v^{\prime} / \partial y\right)_{w}^{2}} v_{w}^{2} / u_{\tau}^{4}$ and $\overline{c_{v}^{2}} v_{w}^{4} / 4 u_{\tau}^{6}=\overline{\left(\partial^{2} v^{\prime} / \partial y^{2}\right)_{w}^{2}} v_{w}^{4} / 4 u_{\tau}^{6}$ using the derivatives from DNS data. Consistent with the behaviour of the exponents, we see that at low and high Mach numbers, the value of the prefactor tends to the $c_{v}$ and $b_{v}$, respectively. Note that $\overline{b_{v}^{2}} v_{w}^{2} / u_{\tau}^{4}$, which we will denote by $\left(\theta_{r m s}^{+}\right)^{2}$, is the variance of dilatation in inner scales and, thus, a proper metric for compressibility effects at the wall. As seen from the figure, around the transition, the magnitude of that term increases substantially as $M_{c}$ is increased. At $M_{c}>0.5$, the prefactor $\sigma_{v v}$ follows the variance of dilatation at the wall.

The best-fit exponents for $R_{u v}$ and $R_{v T}$ in $R 1$ are shown in figures $4(c)$ and $4(d)$, respectively, as blue curves. The behaviour is similar to $R_{v v}$ : at low and high values of $M_{c}$, both exponents tend to 3 and 2, respectively, as expected (table 3). However, the transition appears to be much smoother with exponents reaching their theoretical compressible values only at the highest Mach number in the database $\left(M_{c}>2\right)$.

The exponents $\gamma_{\alpha \beta}$ in the second region, $R 2$, are also included in figures $4(a), 4(c)$ and $4(d)$ as red lines. In general, we see that Reynolds stresses in $R 2$ are less susceptible to Mach number effects as compared to those in $R 1$. At low $M_{c}$, the exponents tend to 

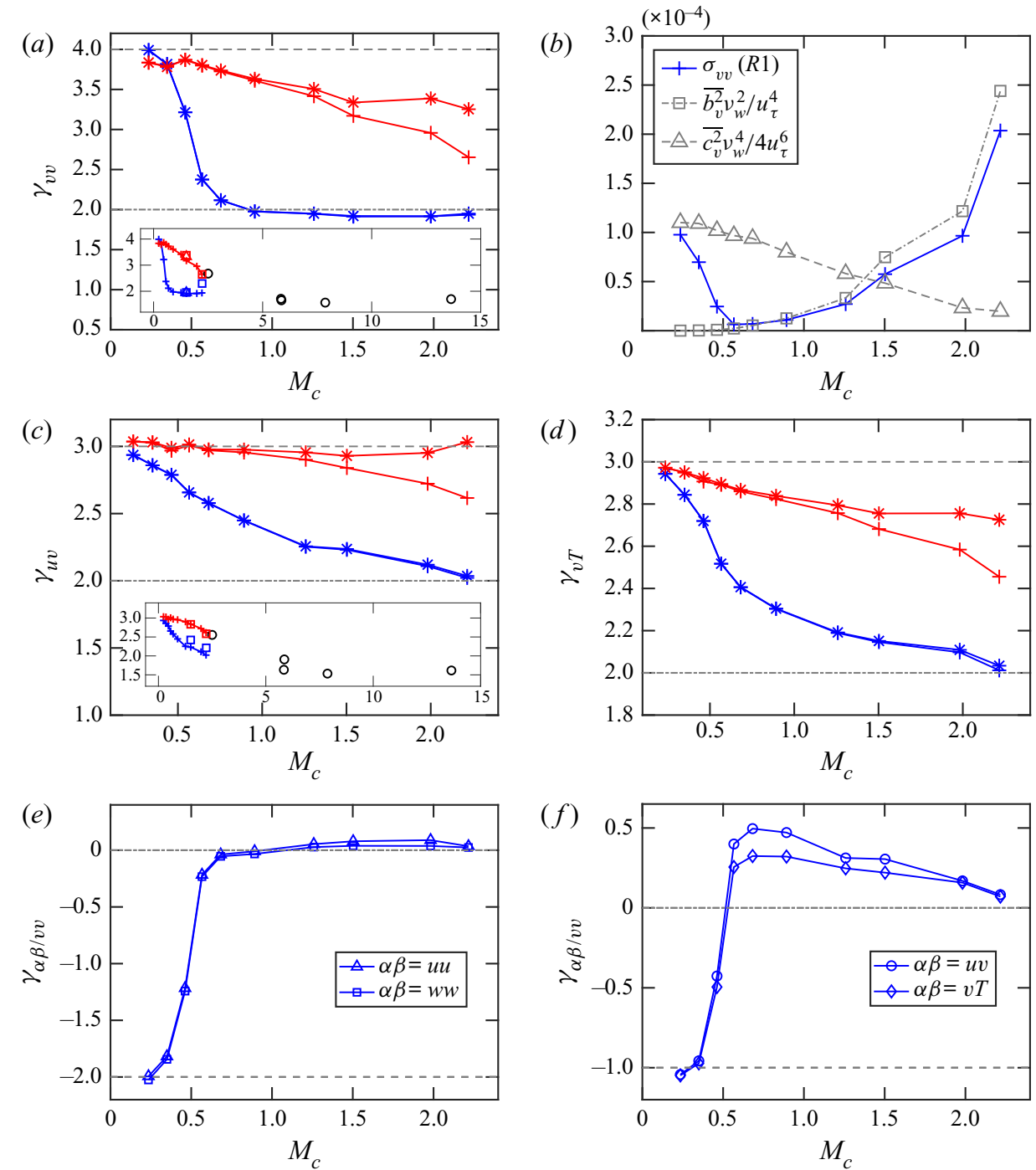

Figure 4. $(a, c-f)$ Power law exponents for fluxes plotted against centreline Mach number. Horizontal lines for solenoidal (dashed) and non-solenoidal (dash-dotted) asymptotic exponents (table 3). Insets in panels (a,c) show exponents from Morinishi et al. (2004) ( $\triangle$ ), Coleman et al. (1995) ( $\square$ ) and Zhang, Duan \& Choudhari (2018) (O). (b) Prefactor $\sigma_{v v}$ and normalized coefficients in Taylor expansion for $R_{v v}$ against $M_{c}$. Markers in panels $(a-d)$ : + indicates wall normalizations $\left(R_{\alpha \beta}=\sigma_{\alpha \beta}\left(y^{+}\right)^{\gamma_{\alpha \beta}}\right)$; $*$ indicates semi-local normalizations $\left(R_{\alpha \beta}^{*}=\sigma_{\alpha \beta}\left(y^{*}\right)^{\gamma_{\alpha \beta}}\right)$. Blue and red markers correspond to $R 1$ and $R 2$ regions, respectively in all panels.

the same values as in $R 1$, which are equal to the theoretical incompressible limit. This reflects the observation that at low Mach numbers, there is simply one scaling range with exponents given by table 3 . The differences in scaling exponents can be linked to different physical processes in the two regimes. In $R 1$, the flow is strongly influenced by viscous effects while in $R 2$, viscous effects are weaker and the flow physics starts being dominated by the interaction of turbulent production and dissipation characteristic of the buffer layer. Because Mach number effects are expected to be different on these processes, it may not be completely surprising that two scaling regimes develop. We also see that scaling 


\section{A. Baranwal, D.A. Donzis and R.D.W. Bowersox}

exponents for wall and semi-local units differ as the Mach number increases. This can be understood by noting that close to the wall (in $R 1$ ), there is little difference between wall and local density and viscosity. In such a case, wall and semi-local variables are practically indistinguishable. This is not the case in $R 2$, where density and viscosity can be very different from $\rho_{w}$, especially as $M_{c}$ increases. This is indeed consistent with the results observed in figures $4(a), 4(c)$ and $4(d)$.

In the insets of figure 4(a,c), we show, along with our results, the data from the supersonic channel of Morinishi et al. (2004) $(\triangle)$ and Coleman et al. (1995) ( $\square$ ). We used the same procedure to find the exponents as that described above. Their exponents exhibit good agreement with our data, especially for $R_{v v}$ in $R 1$ for the case $M_{c}=1.5$, which is expected given that their wall-normal resolution is sufficiently high $\left(y_{w}^{+}=0.1\right)$ and comparable to that used in our simulations at similar conditions. We also include exponents corresponding to supersonic and hypersonic turbulent boundary layers in Zhang et al. (2018) ( $\triangle$ ). The resolutions in that study are insufficient to capture $R 1$; instead, we see their exponents appear to agree with our data in $R 2$ (red symbols). These observations suggest that care must be exercised if asymptotic behaviour is sought but the resolution is not sufficient. Still, it is interesting that the exponents in the channels presented here, seem to agree quantitatively with those in boundary layers, pointing to a potential universal transitional behaviour of high-speed wall-bounded flows. However, we note that there are only very few cases for comparison and a definitive answer will require more data from highly resolved boundary layers.

One can also look at exponents for the ratio of Reynolds stresses $\left(R_{\alpha \beta} / R_{v v}=\right.$ $\sigma_{\alpha \beta / v v} y^{\gamma_{\alpha \beta / v v}}$. This is important as turbulence modelling parameters are often expressed as a function of these ratios (Bowersox 2009). In figure 4(e-f), we show the exponents of these ratios. While the exponents for the ratios of normal stresses (i.e. $R_{u u} / R_{v v}$ and $R_{w w} / R_{v v}$ ) approach their asymptotic behaviour of zero (dashed horizontal line) for $M_{c}$ around unity, there is an overshoot in the exponent for the off-diagonal stresses $\left(R_{u v} / R_{v v}\right.$ and $R_{v T} / R_{v v}$ ) before approaching the theoretical asymptote. This can be explained by noting the slower trend of $R_{u v}$ and $R_{v T}$ to the asymptotic behaviour compared with $R_{v v}$ as $M_{c}$ increases.

We introduce a transition distance from the wall, $y_{t r}$, as the location where the scaling exponents change, that is, the switching point between $R 1$ to $R 2$. This is easily computed with the fits obtained from the DNS data. An example is shown in figure $3(f)$. The transition distance for cases with $M_{c}>0.5$ in our database (cases at lower $M_{c}$ present incompressible scaling over the entire scaling range and, thus, no transition can be meaningfully discerned) and for different quantities is seen in figure 5(a) as a function of $M_{c}$. Apparently, $y_{t r}$ moves away from the wall when compressibility levels are increased. It is interesting that $R_{v v}$ transitions to the near-wall asymptotic behaviour further away from the wall than the off-diagonal stresses and heat flux. We also calculated the transition from the data in Morinishi et al. (2004) and Coleman et al. (1995) and, as seen in figure 5(a), these are in close agreement with our data. As discussed earlier, the other diagonal stresses (i.e. $R_{11}$ and $R_{33}$ ) exhibit a single power law throughout the near-wall region and there is, therefore, no transition. Capturing these different behaviours is important for both turbulence modelling to exhibit the proper asymptotic behaviour in models and in simulations or experiments to determine appropriate resolution requirements. At present, no theoretical result is available to predict $y_{t r}$. However, we found that the data can be fitted with stretched exponentials in $M_{c}$ (included in figure 5(a) and noted in its caption), which may be useful for turbulence modelling and design of numerical simulations. 

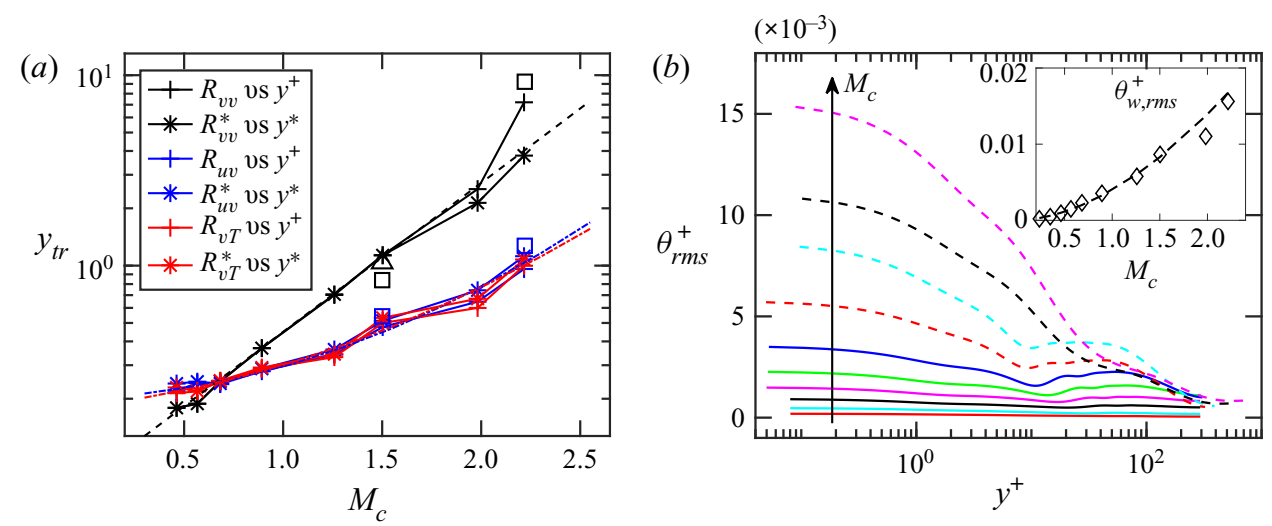

Figure 5. (a) Transition location of scaling exponents. Data from Morinishi et al. (2004) $\left(\triangle, R_{v v}\right)$ and Coleman et al. (1995) ( $\square, R_{v v}$; blue $\square, R_{u v}$ ) in wall units for comparison; best fits of $y_{t r}^{*}$ with $M_{c}$ for $R_{v v}^{*}$, $R_{u v}^{*}$ and $R_{v T}^{*}$ respectively $y_{t r, v v}^{*} \approx \exp \left(1.8 M_{c}-2.6\right.$ ) (dashed), $y_{t r, u v}^{*} \approx \exp \left(0.29 M_{c}^{2}+0.093 M_{c}-1.6\right)$ (blue dashed) and $y_{t r, v T}^{*} \approx \exp \left(0.22 M_{c}^{2}+0.283 M_{c}-1.7\right)$ (red dashed). (b) Distribution of variance of dilatation. The inset has dilatation at the wall $(\diamond)$ and scaling, $0.004 M_{c}^{1.8}$ (dashed).

Finally, we comment on the validity of Morkovin's hypothesis and semi-local scaling close to the wall. The lack of collapse in $R 1$ seen in figure 3 , except for $R_{u u}$, suggests that Morkovin's scaling does not incorporate all compressibility effects associated with increasing the Mach number. Specifically, the hypothesis suggests that compressibility effects can be accounted for by variations in mean density and effects arising from dilatation motions are negligible except at hypersonic speeds. However, our results above show that the fluctuating dilatation at the wall is the main reason for different wall asymptotics. In figure 5(b), we show the root-mean-square dilatation, $\theta_{r m s}^{+}$as a function of $y^{+}$. As noted in the literature (Yu et al. 2019), dilatation fluctuations grow as one approaches the wall. At the wall, $\theta_{w, r m s}^{+}$increases rapidly with the centreline Mach number. This is seen in the inset of the figure, where we also show that this growth is well represented by $\theta_{w, r m s}^{+} \sim M_{c}^{1.8}$. This expression is interesting, as it connects directly the behaviour of compressible motions at the wall with the Mach number very far from the wall. This long-range coupling may cast doubt on simple rescaling approximations such as Morkovin's very close to the wall. However, we also see in figure $4(c)$ that in $R 2, \gamma_{u v}$ is indeed approximately constant in semi-local units, though not for the other exponents.

\section{Conclusions}

The asymptotic behaviour of turbulent stresses and heat flux are investigated close to the wall and compressibility effects are discussed. We present our results with a large database of DNS, with Mach number ranging from incompressible channel centreline Mach number, $M_{c}=0.23$ to a high Mach number, $M_{c}=2.2$. All of our simulations are run with very high near-wall resolution in the wall-normal direction, which we show is essential to capture correct near-wall asymptotic turbulent behaviour. Power laws are fit to the fluxes containing wall-normal velocity fluctuations. Our focus was to capture the variations of the power-law exponents with centreline Mach number changes. Our data suggest that with increasing Mach number, the near-wall asymptotic power-law exponents undergo a smooth transition from the incompressible limit dictated by the solenoidal nature of the velocity field to the power law expected from a general Taylor 


\title{
A. Baranwal, D.A. Donzis and R.D.W. Bowersox
}

series expansion. This behaviour is explained by the degree of dilatation at the wall, which increases with centreline Mach number. We conclude that these dilatational effects, which are generally neglected indeed alter the near-wall flow dynamics and may need to be accounted for in models. Furthermore, beyond this asymptotic region (which we call $R 1$ ), we found a second scaling region $(R 2)$ with a steeper exponent which presents a weaker Mach number effect. However, the transition between $R 1$ and $R 2$ was found to depend strongly on Mach number, even when the asymptotic exponents in $R 1$ attained their theoretical limiting behaviour. We have also found that $R_{v v}$ transitions more quickly and attains its compressible asymptote at a lower Mach number than the cross-correlations $R_{u v}$ and $R_{v T}$. This is also the case for exponents corresponding to ratio of stresses, whose near-wall behaviour is needed in some models. However, higher Mach numbers are needed to assess confidently the approach of some of the fluxes to their corresponding high-Mach-number asymptotes.

A potentially important extension of this work is related to unsteady effects. In a recent study (Saavedra, Poggie \& Paniagua 2020), it was found that free-stream acceleration does affect the near-wall region, especially wall fluxes, when the free stream was accelerated from subsonic to supersonic speeds. It would be interesting to assess the behaviour of scaling exponents in unsteady situations and other more general conditions including, for example, pressure gradients. This may also require further studies on appropriate boundary conditions for these flows.

Funding. The authors gratefully acknowledge support for this work in part from a grant from the Department of Defense Office of the Under Secretary of Defense (Vannevar Bush Faculty Fellowship Grant N00014-18-1-3020). Its contents are solely the responsibility of the authors and do not necessarily represent the official views of the Department of Defense Office of the Under Secretary of Defense. The authors acknowledge the Extreme Science and Engineering Discovery Environment (XSEDE) and Texas Advanced Computing Center (TACC) for computational resources.

Declaration of interests. The authors report no conflict of interest.

\author{
Author ORCIDs. \\ Akanksha Baranwal https://orcid.org/0000-0002-8272-1738; \\ Diego A. Donzis https://orcid.org/0000-0002-1008-221X; \\ Rodney D.W. Bowersox https://orcid.org/0000-0003-4849-8293.
}

\section{REFERENCES}

Bowersox, R.D.W. 2009 Extension of equilibrium turbulent heat flux models to high-speed shear flows. J. Fluid Mech. 633, 61-70.

Coleman, G.N., Kim, J. \& Moser, R.D. 1995 A numerical study of turbulent supersonic isothermal-wall channel flow. J. Fluid Mech. 305, 159-183.

DuAn, L., BeEkMAN, I. \& MARTin, M.P. 2011 Direct numerical simulation of hypersonic turbulent boundary layers. Part 3. Effect of Mach number. J. Fluid Mech. 672, 245-267.

Durbin, P.A. 1993 A Reynolds stress model for near-wall turbulence. J. Fluid Mech. 249, 465-498.

FOYSI, H., SARKAR, S. \& FRIEDRICH, R. 2004 Compressibility effects and turbulence scalings in supersonic channel flow. J. Fluid Mech. 509, 207-216.

Gerolymos, G.A. \& VAllet, I. 2014 Pressure, density, temperature and entropy fluctuations in compressible turbulent plane channel flow. J. Fluid Mech. 757, 701-746.

HinZE, J.O. 1975 Turbulence, 2nd edn. McGraw Hill.

Huang, P.G., Coleman, G.N. \& Bradshaw, P. 1995 Compressible turbulent channel flows: DNS results and modelling. J. Fluid Mech. 305, 185-218.

LAI, Y.G. \& So, R.M.C. 1990 Near-wall modeling of turbulent heat fluxes. Intl J. Heat Mass Transfer 33 (7), 1429-1440. 


\section{Wall asymptotics in compressible turbulent channels}

Li, W., Fan, Y., Modesti, D. \& Cheng, C. 2019 Decomposition of the mean skin-friction drag in compressible turbulent channel flows. J. Fluid Mech. 875, 101-123.

Modesti, D. \& Pirozzoli, S. 2016 Reynolds and mach number effects in compressible turbulent channel flow. Intl J. Heat Fluid Flow 59, 33-49.

Morinishi, Y., TAMAnO, S. \& NAKABAYAShi, K. 2004 Direct numerical simulation of compressible turbulent channel flow between adiabatic and isothermal walls. J. Fluid Mech. 502, 273-308.

Patel, A., Peeters, J.W.R., Boersma, B.J. \& PeCnik, R. 2015 Semi-local scaling and turbulence modulation in variable property turbulent channel flows. Phys. Fluids 27 (9), 095101-1-24.

SaAvedra, J., Poggie, J. \& Paniagua, G. 2020 Response of a turbulent boundary layer to rapid freestream acceleration. Phys. Fluids 32 (4), 045105.

Sciacovelli, L., Cinnella, P. \& Gloerfelt, X. 2017 Direct numerical simulations of supersonic turbulent channel flows of dense gases. J. Fluid Mech. 821, 153-199.

Smits, A.J. \& Dussauge, J.P. 2006 Turbulent Shear Layers in Supersonic Flow. Springer.

So, R.M.C., GATSKi, T.B. \& Sommer, T.P. 1998 Morkovin hypothesis and the modeling of wall-bounded compressible turbulent flows. AIAA J. 36 (9), 1583-1592.

So, R.M.C., LAI, Y.G., ZHANG, H.S. \& HwANG, B.C. 1991a Second-order near-wall turbulence closures a review. AIAA J. 29 (11), 1819-1835.

So, R.M.C., Zhang, H.S. \& Speziale, C.G. $1991 b$ Near-wall modeling of the dissipation rate equation. AIAA J. 29 (12), 2069-2076.

SOMmer, T.P., So, R.M.C. \& ZHANG, H.S. 1993 Near-wall variable-Prandtl-number turbulence model for compressible flows. AIAA J. 31 (1), 27-35.

TretTEL, A. \& LARSSON, J. 2016 Mean velocity scaling for compressible wall turbulence with heat transfer. Phys. Fluids 28 (2), 026102.

Wu, M. \& MARTIN, M.P. 2007 Direct numerical simulation of supersonic turbulent boundary layer over a compression ramp. AIAA J. 45 (4), 879-889.

Yu, M., Xu, C. \& Pirozzoli, S. 2019 Genuine compressibility effects in wall-bounded turbulence. Phys. Rev. Fluids 4, 123402.

Zhang, C., DuAn, L. \& Choudhari, M.M. 2018 Direct numerical simulation database for supersonic and hypersonic turbulent boundary layers. AIAA J. 56 (11), 4297-4311.

Zhang, H., So, R., Speziale, C. \& LAI, Y. 1992 A near-wall two-equation model for compressible turbulent flows. In AIAA, 30th Aerospace Sciences Meeting and Exhibit. AIAA paper 92-0442. 